\title{
ON PRIME NUMBERS OF REAL QUADRATIC FIELDS IN RECTANGLES*
}

\author{
BY \\ HANS RADEMACHER
}

In a recently published paper $\dagger \mathrm{I}$ investigated the number $P\left(x, x^{\prime}\right)$ of primes $\omega$ in a real quadratic field satisfying the inequalities $0<\omega<x$, $0<\omega^{\prime}<x^{\prime}$. The method used there depends on certain refined estimates of the "angular" distribution of prime numbers in real quadratic fields. $\ddagger$ In the present paper I propose to give another more direct proof for the estimate of $P\left(x, x^{\prime}\right)$, which starts from the obvious remark that for a totally positive unit $\eta$ of the field we have

$$
P\left(x, x^{\prime}\right)=P\left(x \eta, x^{\prime} \eta^{\prime}\right) .
$$

This fact leads to the periodicity of the function $P\left(x \eta^{v}, x^{\prime} \eta^{\prime v}\right)$ with respect to the variable $v$, and subsequently to a Fourier development of $P\left(x \eta^{v}, x^{\prime} \eta^{\prime v}\right)$ as function of $v . \S$

Our function $P\left(x, x^{\prime}\right)$ is a special case of a more general type, which may be described as follows: Let $f\left(\mu, \mu^{\prime}\right)$ be a function defined for all integers $\mu$ of the real quadratic field $k$ ( $\mu^{\prime}$ being the conjugate of $\mu$ ) having the property

$$
f\left(\mu, \mu^{\prime}\right)=f\left(\mu \eta, \mu^{\prime} \eta^{\prime}\right)
$$

for all totally positive units $\eta$ of the field. Then we have

$$
\begin{aligned}
F\left(x, x^{\prime}\right) & =\sum_{\substack{0<\mu<x \\
0<\mu^{\prime}<x^{\prime}}} f\left(\mu, \mu^{\prime}\right)=\sum_{\substack{0<\mu \eta<x \eta \\
0<\mu^{\prime} \eta^{\prime}<x^{\prime} \eta^{\prime}}} f\left(\mu, \mu^{\prime}\right)=\sum_{\substack{0<\mu \eta<x \eta \\
0<\mu^{\prime} \eta^{\prime}<x^{\prime} \eta^{\prime}}} f\left(\mu \eta, \mu^{\prime} \eta^{\prime}\right) \\
& =\sum_{\substack{0<\nu<x \eta \\
0<\nu^{\prime}<x^{\prime} \eta^{\prime}}} f\left(\nu, \nu^{\prime}\right)=F\left(x \eta, x^{\prime} \eta^{\prime}\right),
\end{aligned}
$$

analogous to (1). But $F\left(x, x^{\prime}\right)$ is of course discontinuous and hence would not furnish an absolutely convergent Fourier series, which however we need

* Presented to the Society, October 26, 1935; received by the editors October 4, 1935.

†Über die Anzahl der Primzahlen eines reell-quadratischen Zahlkörpers, deren Konjugierte unterhalb gegebener Grenzen liegen, Acta Arithmetica, vol. 1 (1935), pp. 67-77, subsequently cited as "K."

$\ddagger$ Primzahlen reell-quadratischer Zahlkörper in Winkelräumen, Mathematische Annalen, vol. 111 (1935), pp. 209-228, subsequently referred to as "W."

\$ My attention was drawn to this use of the Fourier development by a remark of Siegel, who, as I have heard from him, some years ago found an identity similar to formula (13). In my former method the Fourier expansion was used at another step of the proof, viz., in connection with the angular distribution of the primes. 
for the application to the estimate of the number of primes in certain rectangles $(\$ 2)$. We therefore prefer to investigate

$$
F_{1}\left(x, x^{\prime}\right)=\sum_{0-3 \mu-3 x}(x-\mu)\left(x^{\prime}-\mu^{\prime}\right) f\left(\mu, \mu^{\prime}\right)
$$

(the notation $0-3 \mu-3 x$ being an abbreviation of both the inequalities $0<\mu<x$ and $0<\mu^{\prime}<x^{\prime}$ together), which yields an absolutely convergent Fourier series.

In $\S 2$ we specialize $f\left(\mu, \mu^{\prime}\right)$ and hence $F_{1}\left(x, x^{\prime}\right)$ for our prime-number problem and then have to make use of some results given in "W" concerning Hecke's $\zeta(s, \lambda)$-functions. In $\S 3$ we are in a position to go back from $F_{1}\left(x, x^{\prime}\right)$ to $F\left(x, x^{\prime}\right)$ in this special case. Our principal results are the formulas (13), (33), (43).

For the sake of simplicity we content ourselves with these formulas. Of course no fundamental changes would occur if we introduce an ideal modul $\mathfrak{a}$ and a fixed algebraic integer $\kappa$ and then sum only over the integers $\mu \equiv \kappa$ $(\bmod a)$. Instead of the totally positive fundamental unit $\eta$ we should have to use the totally positive fundamental unit $\eta_{\mathfrak{a}} \bmod \mathfrak{a}$, i.e., with $\eta_{\mathfrak{a}} \equiv 1(\bmod \mathfrak{a})$. But the more general result having been already given as a theorem of " $\mathrm{K}$," page 76, and our main interest being at present the exhibition of the other method, we confine ourselves to the case $\mathfrak{a}=(1)$.

\section{A Fourier expansion}

Let $\eta>0$ be the totally positive fundamental unit, and especially $\eta>1$; we then have

$$
\eta^{\prime}>0, \quad \eta \eta^{\prime}=1, \quad \eta^{\prime}=\eta^{-1} .
$$

Let $f\left(\mu, \mu^{\prime}\right)$ have the property (2). Then we build up the function

$$
F_{1}\left(x, x^{\prime}\right)=\sum_{0-3 \mu-3 x}(x-\mu)\left(x^{\prime}-\mu^{\prime}\right) f\left(\mu, \mu^{\prime}\right) .
$$

Now we have

$$
\begin{aligned}
F_{1}\left(x \eta, x^{\prime} \eta^{\prime}\right) & =\sum_{0-3 \mu-3 x \eta}(x \eta-\mu)\left(x^{\prime} \eta^{\prime}-\mu^{\prime}\right) f\left(\mu, \mu^{\prime}\right) \\
& =\sum_{0-3 \nu \eta-3 x \eta}(x \eta-\nu \eta)\left(x^{\prime} \eta^{\prime}-\nu^{\prime} \eta^{\prime}\right) f\left(\nu \eta, \nu^{\prime} \eta^{\prime}\right) \\
& =\sum_{0-3 \nu-3 x}(x-\nu)\left(x^{\prime}-\nu^{\prime}\right) f\left(\nu, \nu^{\prime}\right)=F_{1}\left(x, x^{\prime}\right) .
\end{aligned}
$$

Therefore $F_{1}\left(x \eta^{v}, x^{\prime} \eta^{-v}\right)$ as function of $v$ is periodic with the period 1. It has obviously a bounded derivative and therefore can be developed into an ab- 
solutely convergent Fourier series, which we write down immediately for the special value $v=0$ :

$$
\begin{aligned}
F_{1}\left(x, x^{\prime}\right) & =\sum_{n=-\infty}^{+\infty} \int_{0}^{1} e^{-2 \pi i n v} F_{1}\left(x \eta^{v}, x^{\prime} \eta^{-v}\right) d v \\
& =\sum_{n=-\infty}^{+\infty} \int_{0}^{1} e^{-2 \pi i n v} \sum_{\substack{0<\mu<x \eta^{v} \\
0<\mu^{\prime}<x^{\prime} \eta^{-v}}}\left(x \eta^{v}-\mu\right)\left(x^{\prime} \eta-v-\mu^{\prime}\right) f\left(\mu, \mu^{\prime}\right) d v .
\end{aligned}
$$

If we collect associated numbers, i.e., numbers differing only by factors that are powers of $\eta,{ }^{*}$ we get

$$
=\sum_{n=-\infty}^{+\infty} \int_{0}^{1} e^{-2 \pi i n v} \sum_{\substack{(\mu) \\ \mu \ell-0 \\ N(\mu)<x x^{\prime}}} f\left(\mu, \mu^{\prime}\right) \sum_{\substack{k \\ 0<\mu k<<\eta^{v} \\ 0<\mu^{\prime} \eta^{-k}<x^{\prime} \eta^{-v}}}\left(x \eta^{v}-\mu \eta^{k}\right)\left(x^{\prime} \eta^{-v}-\mu^{\prime} \eta^{-k}\right) d v,
$$

where the notation $(\mu)_{1}$ indicates that only one representative $\mu$ is taken out of each set of associated numbers (in the narrowest sense). The finite summation over $(\mu)_{1}$ may at once be interchanged with the integration, and in the exponential function we can replace $v$ by $v-k$ :

$$
=\sum_{n=-\infty}^{+\infty} \sum_{\substack{(\mu)_{1} \\ \mu \varepsilon-0 \\ N(\mu)<x x^{\prime}}} f\left(\mu, \mu^{\prime}\right) \int_{0}^{1} \sum_{\substack{k \\ 0<\eta^{k}<x \eta^{v} \\ 0<\mu^{\prime} \eta^{-k}<x^{\prime} \eta^{-v}}} e^{-2 \pi i n(v-k)}\left(x \eta^{v}-\mu \eta^{k}\right)\left(x^{\prime} \eta^{-v}-\mu^{\prime} \eta^{-k}\right) d v .
$$

If we now interchange also the integration and the summation with respect to $k$, we observe that $v$ is not only bounded by $0 \leqq v \leqq 1$, but for each $k$ also by $\mu \eta^{k}<x \eta^{v}$ and by $\mu^{\prime} \eta^{-k}<x^{\prime} \eta^{-v}$, which we can express as follows:

$$
F_{1}\left(x, x^{\prime}\right)=\sum_{n=-\infty}^{+\infty} \sum_{\substack{(\mu)_{1} \\ \mu \in 0 \\ N(\mu)<x x^{\prime}}} f\left(\mu, \mu^{\prime}\right) \sum_{k} \int e^{-2 \pi i n(v-k)}\left(x \eta^{v-k}-\mu\right)\left(x^{\prime} \eta^{-v+k}-\mu^{\prime}\right) d v,
$$

the range of integration in $v$ for each $k$ being determined by the two conditions

$$
-k \leqq v-k \leqq 1-k, \quad \frac{\mu}{x}<\eta^{v-k}<\frac{x^{\prime}}{\mu}
$$

only those values of $k$ are admitted for which the second condition yields a $v$ between 0 and 1 . We substitute $w$ for $v-k$ :

* More precisely these numbers should be called "associated in the narrowest sense," as only totally positive units and not all units are admitted as factors. We shall have to recall this distinction later, on p. 387. 


$$
F_{1}\left(x, x^{\prime}\right)=\sum_{-\infty}^{+\infty} \sum_{\substack{(\mu)_{1} \\ \mu \in 0 \\ N(\mu)<x x^{\prime}}} f\left(\mu, \mu^{\prime}\right) \sum_{k} \int e^{-2 \pi i n w}\left(x \eta^{w}-\mu\right)\left(x^{\prime} \eta^{-w}-\mu^{\prime}\right) d w,
$$

with the conditions of integration

$$
-k \leqq w \leqq 1-k, \quad \frac{\log \frac{\mu}{x}}{\log \eta}<w<\frac{\log \frac{x^{\prime}}{\mu^{\prime}}}{\log \eta} .
$$

But $k$ running through all appropriate integers, the integrals for successive $k$ unite to one integral, the boundaries of which are given by the second condition alone:

$$
\begin{aligned}
F_{1}\left(x, x^{\prime}\right) & =\sum_{n=-\infty}^{+\infty} \sum_{\substack{(\mu)_{1} \\
\mu \& 0 \\
N(\mu)<x x^{\prime}}} f\left(\mu, \mu^{\prime}\right) \int_{\log (\mu / x) / \log \eta}^{\log \left(x^{\prime} / \mu^{\prime}\right) / \log \eta} e^{-2 \pi i n w}\left(x \eta^{w}-\mu\right)\left(x^{\prime} \eta^{-w}-\mu^{\prime}\right) d w \\
& =\sum_{n=-\infty}^{+\infty} \sum_{\substack{(\mu)_{1} \\
\mu \varepsilon 0 \\
N(\mu)<x x^{\prime}}} f\left(\mu, \mu^{\prime}\right) I_{n}\left(\mu, \mu^{\prime}\right)
\end{aligned}
$$

say. We have now to evaluate the integrals $I_{n}\left(\mu, \mu^{\prime}\right)$.

First, for $n \neq 0$, we get

$$
\begin{aligned}
& I_{n}\left(\mu, \mu^{\prime}\right)=\int_{\log (\mu / x) / \log \eta}^{\log \left(x^{\prime} / \mu^{\prime}\right) / \log \eta}\left\{\left(x x^{\prime}+\mu \mu^{\prime}\right) e^{-2 \pi i n w}-\mu x^{\prime} e^{-2 \pi i n w-w \log \eta}\right. \\
& \left.-\mu^{\prime} x e^{-2 \pi i n w+w \log \eta}\right\} d w \\
& =\left(\frac{x^{\prime}}{\mu^{\prime}}\right)^{-2 \pi i n / \log \eta}\left\{-\frac{x x^{\prime}+N(\mu)}{2 \pi i n}+\frac{N(\mu)}{2 \pi i n+\log \eta}+\frac{x x^{\prime}}{2 \pi i n-\log \eta}\right\} \\
& +\left(\frac{\mu}{x}\right)^{-2 \pi i n / \log \eta}\left\{\frac{x x^{\prime}+N(\mu)}{2 \pi i n}-\frac{x x^{\prime}}{2 \pi i n+\log \eta}-\frac{N(\mu)}{2 \pi i n-\log \eta}\right\} \\
& =\frac{\log \eta}{2 \pi i n}\left(\frac{x}{x^{\prime}}\right)^{\pi i n / \log \eta}\left(x x^{\prime}\right)^{-\pi i n / \log \eta}\left(\frac{\mu}{\mu^{\prime}}\right)^{-\pi i n / \log \eta} N(\mu)^{\pi i n / \log \eta} \\
& \cdot\left\{\frac{x x^{\prime}}{2 \pi i n-\log \eta}-\frac{N(\mu)}{2 \pi i n+\log \eta}\right\} \\
& +\frac{\log \eta}{2 \pi i n}\left(\frac{x}{x^{\prime}}\right)^{\pi i n / \log \eta}\left(x x^{\prime}\right)^{\pi i n / \log \eta}\left(\frac{\mu}{\mu^{\prime}}\right)^{-\pi i n / \log \eta} N(\mu)^{-\pi i n / \log \eta} \\
& \cdot\left\{\frac{x x^{\prime}}{2 \pi i n+\log \eta}-\frac{N(\mu)}{2 \pi i n-\log \eta}\right\} \text {, }
\end{aligned}
$$


and finally

$$
\begin{aligned}
& I_{n}\left(\mu, \mu^{\prime}\right)=\frac{\log \eta}{2 \pi i n}\left(\frac{x}{x^{\prime}}\right)^{\pi i n / \log \eta}\left(\frac{\mu}{\mu^{\prime}}\right)^{-\pi i n / \log \eta} x x^{\prime} \\
& {\left[\frac{\left(\frac{N(\mu)}{x x^{\prime}}\right)^{\pi i n / \log \eta}-\left(\frac{N(\mu)}{x x^{\prime}}\right)^{1-\pi i n / \log \eta}}{2 \pi i n-\log \eta}\right.} \\
&+\left.\frac{\left(\frac{N(\mu)}{x x^{\prime}}\right)^{-\pi i n / \log \eta}-\left(\frac{N(\mu)}{x x^{\prime}}\right)^{1+\pi i n / \log \eta}}{2 \pi i n+\log \eta}\right] .
\end{aligned}
$$

For $n=0$ we get by an easy calculation

$$
\begin{aligned}
I_{0}\left(\mu, \mu^{\prime}\right) & =\int_{\log (\mu / x) / \log \eta}^{\log \left(x^{\prime} / \mu^{\prime}\right) / \log \eta}\left\{\left(x x^{\prime}+\mu \mu^{\prime}\right)-\mu x^{\prime} e^{-w \log \eta}-\mu^{\prime} x e^{w \log \eta}\right\} d w \\
& =\frac{1}{\log \eta}\left\{\left(x x^{\prime}+N(\mu)\right) \log \frac{x x^{\prime}}{N(\mu)}-2\left(x x^{\prime}-N(\mu)\right)\right\} .
\end{aligned}
$$

Before introducing (5) and (6) into (4) we make use of the following

LEMMA. Let $0<y, y^{s}=y^{e \log y}$ with the principal value of $\log y$, and $\alpha, \beta$ complex numbers, $c$ real with $c>\max (\Re(\alpha), \Re(\beta))$. Then

$$
\frac{1}{2 \pi i} \int_{c-i \infty}^{c+i \infty} \frac{y^{s}}{(s+\alpha)(s+\beta)} d s=\left\{\begin{array}{lr}
0, & 0<y \leqq 1, \\
\frac{y^{-\alpha}}{\beta-\alpha}-\frac{y^{-\beta}}{\beta-\alpha}, & 1 \leqq y .
\end{array}\right.
$$

To prove the lemma we consider first an integral extended from $c-i \Omega$ to $c+i \Omega$ with large positive $\Omega$. This path of integration parallel to the imaginary axis is then to be replaced by a half-circle with radius $\Omega$, center $c$; for $0<y \leqq 1$ we take the half-circle to the right-hand side (side of the positive real part in the $s$-plane), for $1 \leqq y$ we take the half-circle to the left-hand side. An easy estimate shows that on both half-circles the integral tends to zero with infinitely increasing radius $\Omega$. In the first case no pole is enclosed between the new and the old path of integration, in the second the poles $-\alpha$ and $-\beta$. The calculus of residues then yields the result.

An application of $(7)$ to $(5)$ gives for $0<N(\mu) /\left(x x^{\prime}\right)<1$

$$
\begin{aligned}
& I_{n}\left(\mu, \mu^{\prime}\right) \\
& \quad=\frac{1}{2 \pi i n}\left(\frac{x}{x^{\prime}}\right)^{i \gamma n}\left(\frac{\mu}{\mu^{\prime}}\right)^{-i \gamma n} x x^{\prime}\left[-\frac{1}{2 \pi i} \int_{c-i \infty}^{c+i \infty} \frac{\left(\frac{x x^{\prime}}{N(\mu)}\right)^{s}}{(s+i \gamma n)(s+1-i \gamma n)} d s\right.
\end{aligned}
$$




$$
\left.+\frac{1}{2 \pi i} \int_{c-i \infty}^{o+i \infty} \frac{\left(\frac{x x^{\prime}}{N(\mu)}\right)}{(s-i \gamma n)(s+1+i \gamma n)} d s\right]
$$

with $c>0$ and the abbreviation

$$
\gamma=\frac{\pi}{\log \eta} .
$$

The two integrals can be contracted into one:

$$
I_{n}\left(\mu, \mu^{\prime}\right)=\frac{x x^{\prime}}{\log \eta}\left(\frac{x}{x^{\prime}}\right)^{i \gamma n}\left(\frac{\mu}{\mu^{\prime}}\right)^{-i \gamma n}
$$

$$
\frac{1}{2 \pi i} \int_{c-i \infty}^{c+i \infty} \frac{\left(\frac{x x^{\prime}}{N(\mu)}\right)^{s}}{(s+i \gamma n)(s-i \gamma n)(s+1+i \gamma n)(s+1-i \gamma n)} d s .
$$

The definition (4) shows clearly that $I_{n}\left(\mu, \mu^{\prime}\right)$ depends on $n$ continuously. Hence (10) is valid for $n=0$ also. This could of course be verified by direct reference to (6).

By our lemma the integrals in (8), and therefore also the integrals in (10), are equal to zero for $1 \leqq N(\mu) /\left(x x^{\prime}\right)$ or $N(\mu) \geqq x x^{\prime}$. Hence it is unnecessary after the introduction of (10) into (4) to restrict the summation to the range $N(\mu) \leqq x x^{\prime}$, and thus we get

$$
F_{1}\left(x, x^{\prime}\right)=\frac{x x^{\prime}}{\log \eta} \sum_{n=-\infty}^{+\infty}\left(\frac{x}{x^{\prime}}\right)^{i \gamma n} \sum_{\substack{(\mu)_{1} \\ \mu \neq 0}} f\left(\mu, \mu^{\prime}\right)\left(\frac{\mu}{\mu^{\prime}}\right)^{-i \gamma n}
$$

$$
\frac{1}{2 \pi i} \int_{c-i \infty}^{c+i \infty} \frac{\left(\frac{x x^{\prime}}{N(\mu)}\right)^{\prime}}{(s+i \gamma n)(s-i \gamma n)(s+1+i \gamma n)(s+1-i \gamma n)} d s .
$$

Up to this point the function $f\left(\mu, \mu^{\prime}\right)$ has been subject to no other conditions than (2). Let us now assume

$$
f\left(\mu, \mu^{\prime}\right)=O(|N(\mu)| a)
$$

with a certain real $a$. If we then introduce

$$
Z_{n}(s)=\sum_{\substack{(\mu)_{1} \\ \mu \varepsilon 0}} \frac{f\left(\mu, \mu^{\prime}\right)\left(\frac{\mu}{\mu^{\prime}}\right)^{-i \gamma n}}{N(\mu)^{s}},
$$


the series is absolutely convergent for $\Re(s)>a+1$. In this half-plane the function $Z_{n}(s)$ is certainly regular. The number $c>0$, determining the path of integration in (10), can be chosen as greater than $a+1$. Then the interchange of the summation over $\mu$ and the integration is justified. From (11) and (12) we get

$$
\begin{aligned}
F_{1}\left(x, x^{\prime}\right) & =\sum_{0-3 \mu-3 x}(x-\mu)\left(x^{\prime}-\mu^{\prime}\right) f\left(\mu, \mu^{\prime}\right)=\frac{x x^{\prime}}{\log \eta} \sum_{n=-\infty}^{+\infty}\left(\frac{x}{x^{\prime}}\right)^{i \gamma n} \\
\cdot & \frac{1}{2 \pi i} \int_{c-i \infty}^{c+i \infty} \frac{\left(x x^{\prime}\right)^{s} Z_{n}(s)}{(s+i \gamma n)(s-i \gamma n)(s+1+i \gamma n)(s+1-i \gamma n)} d s .
\end{aligned}
$$

If we treat the term $n=0$ separately by using its original form (6), we have

$$
\begin{aligned}
F_{1}\left(x, x^{\prime}\right)= & \frac{1}{\log \eta} \sum_{\substack{(\mu)_{1} \\
\mu \varepsilon 0 \\
N(\mu)<x x^{\prime}}} f\left(\mu, \mu^{\prime}\right)\left\{\left(x x^{\prime}+N(\mu)\right) \log \frac{x x^{\prime}}{N(\mu)}-2\left(x x^{\prime}-N(\mu)\right)\right\} \\
& +\frac{x x^{\prime}}{\log \eta} \sum_{n=-\infty}^{+\infty}\left(\frac{x}{x^{\prime}}\right)^{i \gamma n} \\
& \frac{1}{2 \pi i} \int_{c-i \infty}^{c+i \infty} \frac{\left(x x^{\prime}\right)^{s} Z_{n}(s)}{(s+i \gamma n)(s-i \gamma n)(s+1+i \gamma n)(s+1-i \gamma n)} d s
\end{aligned}
$$

the prime at the summation sign meaning the omission of $n=0$. In formulas (13) and (13a) we have attained the objects of this paragraph. It may here be added that by quite analogous reasoning and calculation we can derive the formula

$$
\begin{aligned}
F_{0}\left(x, x^{\prime}\right) & =\sum_{0-3 \mu-3 x}^{*} f\left(\mu, \mu^{\prime}\right) \\
& =\frac{1}{\log \eta} \sum_{n=-\infty}^{+\infty}\left(\frac{x}{x^{\prime}}\right)^{i \gamma n} \frac{1}{2 \pi i} \int_{c-i \infty}^{c+i \infty} \frac{\left(x x^{\prime}\right)^{s} Z_{n}(s)}{(s+i \gamma n)(s-i \gamma n)} d s,
\end{aligned}
$$

where $\sum^{*}$ indicates a special treatment of the boundary summands: a term $f\left(\mu, \mu^{\prime}\right)$ with $\mu=x, \mu^{\prime}<x^{\prime}$ or with $\mu<x, \mu^{\prime}=x^{\prime}$ is only to be taken into account as $\frac{1}{2} f\left(\mu, \mu^{\prime}\right)$, whereas a term with $\mu=x, \mu^{\prime}=x^{\prime}$ does not count at all. But the series in (14) with summation over $n$ is not absolutely convergent and therefore not suited to our further applications.

I am in the possession of a more general formula, of which (13) and (14) are special cases and which I hope to communicate on another occasion.

\section{Estimate OF PRIME NUMBERS IN RECTANGLES}

We shall now specialize our formula (13) for treating our prime-number problem. Let us put 


$$
f\left(\mu, \mu^{\prime}\right)=\left\{\begin{array}{l}
1 \text { for }(\mu) \text { prime ideal, } \\
0 \text { otherwise }
\end{array}\right.
$$

For this special $f\left(\mu, \mu^{\prime}\right)$ the function $F\left(x, x^{\prime}\right)$ may be called $P_{1}\left(x, x^{\prime}\right)$. In this case we have

$$
Z_{n}(s)=\sum_{\substack{(\omega)_{1} \\ \omega \in 0}} \frac{\left(\frac{\omega}{\omega^{\prime}}\right)^{-i \gamma n}}{N(\omega)^{s}},
$$

$\omega$ running only through prime numbers, i.e., integers, whose principal ideals $(\omega)$ are prime ideals. We are in a position to discuss the function by reducing it to Hecke's well known $\zeta(s, \lambda)$-functions.

For this purpose we introduce following Hecke $\dagger$ "ideal numbers" $\hat{\mu}$, which together with the numbers of the algebraic field $k$ constitute a certain larger realm $B$ in which multiplication, division, and the operation of determining the greatest common divisor are possible without exception, and in which moreover all units belong to the given field $k$. These numbers can be separated into $2^{2} h$ classes under the stipulation that two belong to the same class when and only when their quotient is a totally positive (integral or fractional) algebraic number of the field. The number $h$ is the ordinary class number.

Let $\chi(\hat{\mu})$ be a character of the Abelian class group of order $2^{2} h$; the unit element of this class group being the class of all totally positive algebraic numbers of the field $k$, we have $\chi(\mu)=1$ for $\mu \varepsilon 0$, and especially $\chi(\eta)=1$ for all characters $\chi$. Hence we have

$$
Z_{n}(s)=\frac{1}{4 h} \sum_{\chi} \sum_{(\hat{\omega})_{1}} \frac{\chi(\hat{\omega}) \lambda^{n}(\hat{\omega})}{|N(\hat{\omega})|^{s}}
$$

where we use Hecke's notation

$$
\lambda(\hat{\mu})=\left(\frac{\hat{\mu}}{\hat{\mu}^{\prime}}\right)^{-i \gamma}=\left(\frac{\hat{\mu}}{\hat{\mu}^{\prime}}\right)^{-\pi i / \log \eta}
$$

and $(\hat{\omega})_{1}$ means again that out of each set of ideal numbers associated in the narrow sense we have only to take one representative. But two numbers not associated in the narrow sense may well be associated in the ordinary sense. If we select only non-associated numbers in the ordinary sense, we must consider units $\theta$ which are not narrowly associated:

$$
Z_{n}(s)=\frac{1}{4 h} \sum_{\chi} \sum_{(\hat{\omega})} \frac{\chi(\hat{\omega}) \lambda^{n}(\hat{\omega})}{|N(\hat{\omega})|^{s}} \sum_{(\theta)_{1}} \chi(\theta) \lambda^{n}(\theta) .
$$

$\dagger$ E. Hecke, Eine neue Art von Zetafunktionen und ihre Beziehungen zur Verteilung der Primzahlen, II, Mathematische Zeitschrift, vol. 6 (1920), fp. 11-51. 
The units not associated in the narrow sense form a group of order $2 q$, where $q$ is defined by

$$
\eta=\epsilon^{q}
$$

with $\epsilon>1$ the ordinary fundamental unit. (For $q=1$ we have only +1 and -1 not associated in the narrow sense; for $q=2$ the units $+1,-1,+\epsilon,-\epsilon$.) Obviously $\chi(\theta) \lambda^{n}(\theta)$ is a character of this group of units.

Now we have to distinguish two cases:

(i) $\chi(\theta) \lambda^{n}(\theta)=1$ for all units $\theta$. This occurs for certain $\chi$, which have the property $\chi(\theta)=\lambda^{-n}(\theta)$ for the finite group of not narrowly associated units. Such $\chi$ always exist. Indeed, for given $\lambda^{n}$ they are determined at first only for the subgroup of such classes which contain units. But according to a general property of characters of Abelian groups it is always possible to extend a character given on a subgroup to the total enclosing group. The number of such characters is equal to the index of the subgroup in the total group; in our case therefore $4 h /(2 q)$. For such $\chi$ we have

$$
\sum_{(\theta)_{1}} \chi(\theta) \lambda^{n}(\theta)=2 q=\frac{2 \log \eta}{\log \epsilon}
$$

because of (18). In this case we call (in a slight modification of Hecke's terminology) the product $\chi(\hat{\mu}) \lambda^{n}(\hat{\mu})$ an "angular character for ideals" and use the abbreviated symbol $\chi \lambda^{n}(\hat{\mu})$. In fact because $\chi \lambda^{n}(\theta)=1$ the character $\chi \lambda^{n}(\hat{\mu})$ has the same value for all $\hat{\mu}$ representing the same ideal.

(ii) Not for all units $\theta$ is $\chi(\theta) \lambda^{n}(\theta)=1$. Then $\chi(\theta) \lambda^{n}(\theta)$ is not the principal character for the subgroup of the classes containing units, hence we have

$$
\sum_{(\theta)_{1}} \chi(\theta) \lambda^{n}(\theta)=0 .
$$

In (17) therefore we have only to consider such $\chi$ as give rise to an angular character $\chi^{n}(\hat{\mu})$ for ideals (such $\chi$ exist, as mentioned above, in number $2 h / q$ ). If in a summation over $\chi$ we have to select such $\chi$ in the manner mentioned with regard to $\lambda^{n}$ we will mark it by a subscript $\lambda^{n}$ attached to the summation sign. From (17) and (19) we have

$$
Z_{n}(s)=\frac{\log \eta}{2 h \log \epsilon} \sum_{\chi} \sum^{n} \sum_{(\hat{\omega})} \frac{\chi \lambda^{n}(\hat{\omega})}{|N(\hat{\omega})|^{s}}=\frac{\log \eta}{2 h \log \epsilon} \sum_{x} Z\left(s, \chi \lambda^{n}\right),
$$

say.

On the other hand we have the definition of Hecke's $\zeta(s, \lambda)$-functions:

$$
\zeta\left(s, \chi \lambda^{n}\right)=\sum_{(\hat{\mu})} \frac{\chi^{\lambda^{n}(\hat{\mu})}}{|N(\hat{\mu})|^{s}}=\prod_{(\hat{\omega})}\left(\frac{x \lambda^{n}(\hat{\omega})}{|N(\hat{\omega})|^{s}}\right)^{-1}
$$


valid for $\Re(s)>1$, and hence

$$
\begin{aligned}
\log \zeta\left(s, \chi \lambda^{n}\right) & =\sum_{(\omega)} \sum_{m=1}^{\infty} \frac{1}{m}\left(\frac{\chi \lambda^{n}(\hat{\omega})}{|N(\hat{\omega})|^{s}}\right)^{m} \\
& =\sum_{(\omega)} \frac{\chi \lambda^{n}(\hat{\omega})}{|N(\hat{\omega})|^{*}}+\sum_{(\omega)} \sum_{m=2}^{\infty} \frac{\left(\chi \lambda^{n}(\hat{\omega})\right)^{m}}{m|N(\hat{\omega})|^{m s}} \\
& =Z\left(s, \chi \lambda^{n}\right)+\Xi\left(s, \chi \lambda^{n}\right),
\end{aligned}
$$

say.

Now $\Xi\left(s, \chi \lambda^{n}\right)$ is regular for $\Re(s)>\frac{1}{2}$, since the defining series is absolutely and uniformly convergent for $\Re(s) \geqq \sigma_{0}>\frac{1}{2}$ :

$$
\begin{aligned}
& \left|\sum_{(\omega)} \sum_{m=2}^{\infty} \frac{\left(\chi \lambda^{n}(\hat{\omega})\right)^{m}}{m|N(\hat{\omega})|^{m s}}\right|<\frac{1}{2} \sum_{(\hat{\omega})} \frac{1}{|N(\hat{\omega})|^{2 \sigma_{0}}} \frac{1}{1-\frac{1}{|N(\hat{\omega})|^{\sigma_{0}}}} \\
& \quad<\frac{1}{2} \sum_{(\hat{\omega})} \frac{1}{|N(\hat{\omega})|^{2 \sigma_{0}}} \frac{1}{1-2^{-\sigma_{0}}}<\frac{1}{2-2^{1-\sigma_{0}}} \sum_{\hat{\mu})} \frac{1}{|N(\hat{\mu})|^{2 \sigma_{0}}} \\
& =\frac{1}{2-2^{1-\sigma_{0}}} \zeta\left(2 \sigma_{0}\right),
\end{aligned}
$$

which shows moreover that for $\Re(s) \geqq \sigma_{0}>\frac{1}{2}$ the function $\Xi\left(s, \chi \lambda^{n}\right)$ is bounded. We can write

$$
Z_{n}(s)=\frac{\log \eta}{2 h \log \epsilon} \sum_{x} \log \zeta\left(s, x \lambda^{n}\right)+B_{n}(s),
$$

where $B_{n}(s)$ is bounded for $\Re(s) \geqq \sigma_{0}>\frac{1}{2}$ and all $n$. If we insert (21) and (15) in (13a), we get

$$
\begin{aligned}
& P_{1}\left(x, x^{\prime}\right)= \sum_{0-3 \omega-3 x}(x-\omega)\left(x^{\prime}-\omega^{\prime}\right)=+H \frac{x x^{\prime}}{2 h \log \epsilon} \sum_{n=-\infty}^{+\infty}\left(\frac{x}{x^{\prime}}\right)^{i \gamma n} \\
& \frac{1}{2 \pi i} \sum_{x} \int_{\lambda n}^{2+i \infty} \frac{\left(x x^{\prime}\right)^{s} \log \zeta\left(s, x \lambda^{n}\right)}{(s+i \gamma n)(s-i \gamma n)(s+1+i \gamma n)(s+1-i \gamma n)} d s \\
& \quad+\frac{x x^{\prime}}{\log \eta} \sum_{n=-\infty}^{+\infty}\left(\frac{x}{x^{\prime}}\right)^{i \gamma n} \\
& \quad \frac{1}{2 \pi i} \int_{2-i \infty}^{2+i \infty} \frac{\left(x x^{\prime}\right)^{s} B_{n}(s)}{(s+i \gamma n)(s-i \gamma n)(s+1+i \gamma n)(s+1-i \gamma n)} d s
\end{aligned}
$$

with

(22a) $\quad H=\frac{1}{\log \eta} \sum_{\substack{\omega \omega) \\ \omega \in d \\ N(\omega)<x x^{\prime}}}\left\{\left(x x^{\prime}+N(\omega)\right) \log \frac{x x^{\prime}}{N(\omega)}-2\left(x x^{\prime}-N(\omega)\right)\right\}$. 
The two infinite sums over $n \neq 0$ in (22) are now to be estimated. We begin with the second of these sums, which is easier to handle. In the following the letter $C$ is used for positive constants, not necessarily always the same.

The functions $B_{n}(s)$ being regular and bounded for $\Re(s) \geqq \sigma_{0}>\frac{1}{2}$, we can shift the path of integration to the left up to the abscissa $\frac{3}{4}$. Then we have

$$
\begin{aligned}
& \left|\int_{3 / 4-i \infty}^{3 / 4+i \infty} \frac{\left(x x^{\prime}\right)^{s} B_{n}(s)}{(s+i \gamma n)(s-i \gamma n)(s+1+i \gamma n)(s+1-i \gamma n)} d s\right| \\
& \quad<C\left(x x^{\prime}\right)^{3 / 4} \int_{-\infty}^{+\infty} \frac{d t}{\left(\frac{3}{4}+|t+\gamma n|\right)\left(\frac{3}{4}+|t-\gamma n|\right)\left(\frac{7}{4}+|t+\gamma n|\right)\left(\frac{7}{4}+|t-\gamma n|\right)} .
\end{aligned}
$$

By reason of symmetry the latter integral from $-\infty$ to $+\infty$ can be replaced by twice the integral extended from 0 to $+\infty$; without loss of generality we can further suppose $n>0$ :

$$
\begin{aligned}
\int_{-\infty}^{+\infty}< & \frac{2}{\left(\frac{3}{4}+\gamma n\right)\left(\frac{7}{4}+\gamma n\right)} \int_{0}^{\gamma n} \frac{d t}{\left(\frac{3}{4}+\gamma n-t\right)\left(\frac{7}{4}+\gamma n-t\right)} \\
& +\frac{2}{\left(\frac{3}{4}+2 \gamma n\right)\left(\frac{7}{4}+2 \gamma n\right)} \int_{\gamma n}^{\infty} \frac{d t}{\left(\frac{3}{4}+t-\gamma n\right)\left(\frac{7}{4}+t-\gamma n\right)} \\
< & \frac{5}{2(\gamma n)^{2}} \int_{0}^{\infty} \frac{d t}{\left(\frac{3}{4}+t\right)\left(\frac{7}{4}+t\right)}=\frac{C}{n^{2}} .
\end{aligned}
$$

Hence

$$
\begin{aligned}
& \left|\frac{x x^{\prime}}{\log \eta} \sum_{n=-\infty}^{+\infty}\left(\frac{x}{x^{\prime}}\right)^{i \gamma n} \frac{1}{2 \pi i} \int_{2-i \infty}^{2+i \infty} \frac{\left(x x^{\prime}\right)^{s} B_{n}(s)}{(s+i \gamma n)(s-i \gamma n)(s+1+i \gamma n)(s+1-i \gamma n)} d s\right| \\
& \quad<C\left(x x^{\prime}\right)^{7 / 4} \sum_{n=1}^{\infty} \frac{1}{n^{2}}=C\left(x x^{\prime}\right)^{7 / 4} .
\end{aligned}
$$

For the estimate of the first infinite sum over $n$ in (22) we shall make use of the following

LEMma. There exists an absolute constant $E \geqq 1$ such that in the region (boundaries included) of the plane of the complex variable $s=\sigma+i t$

$$
1-\frac{1}{6000(\log (1+|n|)+\log (1+|t|))} \leqq \sigma \leqq 3, \quad|t| \geqq E,
$$




$$
1-\frac{1}{6000(\log (1+|n|)+\log (1+E))} \leqq \sigma \leqq 3, \quad|t| \leqq E,
$$

the function $\log \zeta\left(s, \chi \lambda^{n}\right)$, for $n \neq 0$, is regular and satisfies respectively the inequalities

$$
\begin{aligned}
\log \zeta\left(s, \chi \lambda^{n}\right) \mid & \leqq C\left(\log ^{2}(1+|n|)+\log ^{2}(1+|t|)\right), & & |t| \geqq E, \\
& \leqq C\left(\log ^{2}(1+|n|)+\log ^{2}(1+E)\right), & & |t| \leqq E .
\end{aligned}
$$

As to the proof, this lemma is only a slight modification of Theorem 6 in my paper "W." We have only to consider $\log \zeta\left(s, \chi \lambda^{n}\right)$ instead of $\left(\zeta^{\prime} / \zeta\right)\left(s, \chi \lambda^{n}\right)$ in that theorem. The Caratheodory-Landau lemma permits then all the necessary conclusions in the proof. Moreover, in the wording of the present lemma we have replaced $\tau(t, n)$ by its definition $\tau(t, n)=(1+|t|)^{2}(1+|n|)^{2}$.

By means of this lemma we are in a position to transform the path of integration from $2-i \infty$ to $2+i \infty$ into the following path $P$ :

$$
\begin{array}{ll}
\sigma=1-\frac{1}{6000(\log (1+|n|)+\log (1-t))}, & t \leqq-E ; \\
\sigma=1-\frac{1}{6000(\log (1+|n|)+\log (1+E))}, & -E \leqq t \leqq E ; \\
\sigma=1-\frac{1}{6000(\log (1+|n|)+\log (1+t))}, & E \leqq t .
\end{array}
$$

We thus have

$$
\begin{aligned}
& \left|\int_{P} \frac{\left(x x^{\prime}\right)^{8} \log \zeta\left(s, x \lambda^{n}\right)}{(s+i \gamma n)(s-i \gamma n)(s+1+i \gamma n)(s+1-i \gamma n)} d s\right| \\
& \leqq C \int_{0}^{E} \frac{\left(x x^{\prime}\right)^{1-1 /(6000(\log (1+E)+\log (1+|n|)))}\left(\log ^{2}(1+E)+\log ^{2}(1+n \mid)\right) \mid}{\left(\frac{1}{2}+|t+\gamma n|\right)\left(\frac{1}{2}+|t-\gamma n|\right)\left(\frac{3}{2}+|t+\gamma n|\right)\left(\frac{3}{2}+|t-\gamma n|\right)} d t \\
& \quad+C \int_{E}^{\infty} \frac{\left(x x^{\prime}\right)^{1-1 /(6000(\log (1+t)+\log (1+|n|)))}\left(\log ^{2}(1+t)+\log ^{2}(1+|n|)\right)}{\left(\frac{1}{2}+|t+\gamma n|\right)\left(\frac{1}{2}+|t-\gamma n|\right)\left(\frac{3}{2}+|t+\gamma n|\right)\left(\frac{3}{2}+|t-\gamma n|\right)} d t \\
& =I_{1}+I_{2},
\end{aligned}
$$

say. Without loss of generality we can again assume $n>0$. We have $I_{1} \leqq C\left(x x^{\prime}\right)^{1-c / \log (1+n)}$

$$
\cdot \log ^{2}(1+n) \int_{0}^{\infty} \frac{d t}{\left(\frac{1}{2}+|t+\gamma n|\right)\left(\frac{1}{2}+|t-\gamma n|\right)\left(\frac{3}{2}+|t+\gamma n|\right)\left(\frac{3}{2}+|t-\gamma n|\right)},
$$

and the same conclusions which led to (23) yield here 


$$
I_{1} \leqq C\left(x x^{\prime}\right)^{1-c / \log (1+n)} \frac{\log ^{2}(1+n)}{n^{2}} .
$$

As to $I_{2}$, we intersect the path of integration at the points

$$
K=\max (E, \gamma n), \quad L=\max \left(E, 2 \gamma n e^{\left(\log x x^{\prime}\right) 1 / 2}\right)
$$

and have

$$
\int_{E}^{\infty}=\int_{E}^{K}+\int_{K}^{L}+\int_{L}^{\infty}=J_{1}+J_{2}+J_{3},
$$

say. We assume always $x x^{\prime} \geqq 1$; it follows that $\left(\log x x^{\prime}\right)^{1 / 2} \geqq 0$ and hence $K \leqq L$. It may be that $E=K$ or $E=K=L$, in which cases $J_{1}=0$, or $J_{1}=J_{2}=0$, respectively.

For $J_{1}$, only $E<K$ remains to be considered. We have

$$
\begin{aligned}
J_{1} & \leqq \int_{E}^{\gamma n} \frac{\left(x x^{\prime}\right)^{1-c /(\log (1+t)+\log (1+n))}\left(\log ^{2}(1+t)+\log ^{2}(1+n)\right)}{\left(\frac{1}{2}+\gamma n+t\right)\left(\frac{1}{2}+\gamma n-t\right)\left(\frac{3}{2}+\gamma n+t\right)\left(\frac{3}{2}+\gamma n-t\right)} d t \\
& <C \frac{\left(x x^{\prime}\right)^{1-c / \log (1+n)} \log ^{2}(1+n)}{\left(\frac{1}{2}+\gamma n\right)\left(\frac{3}{2}+\gamma n\right)} \int_{0}^{\gamma n} \frac{d t}{\left(\frac{1}{2}+\gamma n-t\right)\left(\frac{3}{2}+\gamma n-t\right)} \\
& <C\left(x x^{\prime}\right)^{1-c / \log (1+n)} \frac{\log ^{2}(1+n)}{n^{2}} .
\end{aligned}
$$

This estimate of course is also valid for $E=K$.

Secondly, for $K=L$ we have $J_{2}=0$. If we assume $K<L$ we have

$$
\begin{aligned}
J_{2} & \leqq \int_{\gamma n}^{2 \gamma n \exp \left(\left(\log x x^{\prime}\right) 1 / 2\right)} \frac{\left(x x^{\prime}\right)^{1-c /(\log (1+n)+\log (1+t))}\left(\log ^{2}(1+n)+\log ^{2}(1+t)\right)}{\left(\frac{1}{2}+t+\gamma n\right)\left(\frac{1}{2}+t-\gamma n\right)\left(\frac{3}{2}+t+\gamma n\right)\left(\frac{3}{2}+t-\gamma n\right)} d t \\
& <C \frac{\left(x x^{\prime}\right)^{1-c /\left(\log (1+n)+\left(\log x x^{\prime}\right) 1 / 2\right)}}{\left(\frac{1}{2}+2 \gamma n\right)\left(\frac{3}{2}+2 \gamma n\right)} \int_{\gamma n}^{\infty} \frac{\log ^{2}(1+t)}{\left(\frac{1}{2}+t-\gamma n\right)\left(\frac{3}{2}+t-\gamma n\right)} d t \\
& <\frac{C}{n^{2}}\left(x x^{\prime}\right)^{1-c /\left(\log (1+n)+\left(\log x x^{\prime}\right) 1 / 2\right)} .
\end{aligned}
$$

Finally we have

$$
\begin{aligned}
J_{3} & \leqq \int_{2 \gamma n \exp \left(\left(\log x x^{\prime}\right)^{1 / 2}\right)}^{\infty}<\frac{x x^{\prime}}{(3 \gamma n)^{2}} \int_{2 \gamma n \exp \left(\left(\log x x^{\prime}\right)^{1 / 2}\right)}^{\infty} \frac{\log ^{2}(1+n)+\log ^{2}(1+t)}{(t-\gamma n)^{2}} d t \\
& <C \frac{x x^{\prime}}{n^{2}} \int_{\gamma n \exp \left(\left(\log x x^{\prime}\right)^{1 / 2}\right)}^{\infty} \frac{\log ^{2}(1+t)}{t^{2}} d t<C \frac{x x^{\prime}}{n^{2}} \int_{\gamma n \exp \left(\left(\log x x^{\prime}\right)^{1 / 2}\right)}^{\infty} \frac{d t}{t^{3 / 2}} \\
& <C \frac{x x^{\prime}}{n^{2}} e^{-(1 / 2)\left(\log x x^{\prime}\right)^{1 / 2}} .
\end{aligned}
$$


These estimates together furnish the inequality

(26) $I_{2}=C\left(J_{1}+J_{2}+J_{3}\right)<C x x^{\prime} e^{-c \log x x^{\prime} /\left(\log (1+|n|)+\left(\log x x^{\prime}\right) 1 / 2\right)} \frac{\log ^{2}(1+|n|)}{n^{2}}$.

Collecting our results we get from (22), (23), (24), (25), (26)

$$
\begin{aligned}
& \sum_{0 \rightarrow 3 \omega-3 x}(x-\omega)\left(x^{\prime}-\omega^{\prime}\right) \\
& \quad=H+O\left(\left(x x^{\prime}\right)^{7 / 4}\right)+O\left(x x^{\prime} \sum_{n=1}^{\infty} \frac{\log ^{2}(1+n)}{n^{2}} e^{-c \log x x^{\prime} /\left(\log (1+n)+\left(\log x x^{\prime}\right) 1 / 2\right)}\right) .
\end{aligned}
$$

In order to calculate the sum in the second $O$-term we put

$$
\begin{aligned}
\sum_{n=1}^{\infty} & =\sum_{n=1}^{\left.\left.\operatorname{loxp}\left(\left(\log x x^{\prime}\right)\right)^{1 / 2}\right)\right]^{-1}}+\sum_{\left[\operatorname{loxp}\left(\left(\log x x^{\prime}\right)^{1 / 2}\right)\right]}^{\infty} \\
& <e^{\left.-(c / 2)\left(\log x x^{\prime}\right)\right)^{1 / 2}} \sum_{n=1}^{\infty} \frac{\log ^{2} n}{n^{2}}+\sum_{\left.n=\left[\exp \left(\left(\log x x^{\prime}\right)\right)^{1 / 2}\right)\right]} \frac{\log ^{2} n}{n^{2}} \\
& <C e^{\left.-(c / 2)\left(\log x x^{\prime}\right)\right)^{1 / 2}}+e^{\left.-(1 / 2)\left(\log x x^{\prime}\right)\right)^{1 / 2}} \sum_{n=1}^{\infty} \frac{\log ^{2} n}{n^{3 / 2}}<C e^{\left.-c^{\prime}\left(\log x x^{\prime}\right)\right)^{1 / 2}} .
\end{aligned}
$$

Hence our result reads as follows:

$$
P_{1}\left(x, x^{\prime}\right)=\sum_{0-3 \omega-3 x}(x-\omega)\left(x^{\prime}-\omega^{\prime}\right)=H+O\left(\left(x x^{\prime}\right)^{2} e^{-c\left(\log x x^{\prime}\right) 1 / 2}\right)
$$

with $c$ in a new meaning.

Our next aim is to give a concise formula for $H$. We start with the formulas

$$
\sum_{\substack{(\omega) 1 \\ \omega \in=1 \\ N(\omega)<y}} 1=\frac{q}{2 h} \int_{2}^{y} \frac{d t}{\log t}+O\left(y e^{-c(\log y) 1 / 2}\right)
$$

and

$$
\sum_{\substack{(\omega)_{1} \\ \omega=0 \\ N(\omega)<y}} \log N(\omega)=\frac{q}{2 h} y+O\left(y e^{-c(\log y) 1 / 2}\right)
$$

where $q$ has the same meaning of as in (18). These equations are well known.* The divisor $2 h / q$ indicates the number of classes of ideals, from which in the. summations only one is considered.

If we have the equation

* Edmund Landau, Über Ideale und Primideale in Idealklassen, Mathematische Zeitschrift, vol. 2 (1918), pp. 52-154, Theorem LXXXIII and Theorem LXXXV. 
we can conclude

$$
\sum_{\substack{(\omega)_{1} \\ \omega \in 0 \\ N(\omega)<y}} c_{\omega}=g(y)
$$

$$
\int_{2}^{Y} g(y) d y=\int_{2}^{Y} \sum_{\substack{(\omega)_{1} \\ \omega \notin 0 \\ N(\omega)<y}} c_{\omega} d y=\sum_{\substack{(\omega)_{1} \\ \omega \& 0 \\ N(\omega)<Y}} c_{\omega} \int_{N(\omega)}^{Y} d y=\sum_{\substack{(\omega)_{1} \\ \omega \in 0 \\ N(\omega)<Y}} c_{\omega}(Y-N(\omega)) .
$$

From (30), (28) and (29) we deduce especially for $c_{\omega}=1$ and $c_{\omega}=\log N(\omega)$

$$
\sum_{\substack{(\omega) 1 \\ \text { s\&o } \\ N(\omega)<Y}}(Y-N(\omega))=\frac{q}{2 h} \int_{2}^{Y} d y \int_{2}^{Y} \frac{d t}{\log t}+O\left(\int_{2}^{Y} y e^{-c(\log y) 1 / 2} d y\right),
$$

$$
\sum_{\substack{(\omega)_{1} \\ \omega \in 0 \\ N(\omega)<Y}}(Y-N(\omega)) \log N(\omega)=\frac{q}{2 h} \int_{2}^{Y} y d y+O\left(\int_{2}^{Y} y e^{-c(\log y)^{1 / 2}} d y\right) .
$$

Now we have

$$
\int_{2}^{Y} d y \int_{2}^{y} \frac{d t}{\log t}=\int_{2}^{Y} \frac{d t}{\log t} \int_{t}^{Y} d y=\int_{2}^{Y} \frac{(Y-t) d t}{\log t}
$$

and

$$
\begin{aligned}
\int_{2}^{Y} y e^{-c(\log y) 1 / 2} d y & =\int_{2}^{Y^{1 / 2}}+\int_{Y^{1 / 2}}^{Y}<\frac{Y}{2}+e^{-c((\log Y) / 2)^{1 / 2}} \int_{Y^{1 / 2}}^{Y} y d y \\
& =O\left(Y^{2} e^{\left.-c^{\prime}(\log Y)^{1 / 2}\right)},\right.
\end{aligned}
$$

and therefore

$$
\begin{aligned}
& \sum_{\substack{(\omega)_{1} \\
\omega \in 0 \\
N(\omega)<Y}}(Y-N(\omega))=\frac{q}{2 h} \int_{2}^{Y} \frac{(Y-t) d t}{\log t}+O\left(Y^{2} e^{-c(\log Y)^{1 / 2}}\right), \\
& \sum_{\substack{(\omega)_{1} \\
\omega \in 0 \\
N(\omega)<Y}}(Y-N(\omega)) \log N(\omega)=\frac{q}{2 h} \frac{Y^{2}}{2}+O\left(Y^{2} e^{-c(\log Y) 1 / 2}\right) .
\end{aligned}
$$

These equations together with (28) and (29) yield

$$
\sum_{\substack{(\omega)_{1} \\ \omega \notin 0 \\ N(\omega)<Y}} N(\omega)=\frac{q}{2 h} \int_{2}^{Y} \frac{t d t}{\log t}+O\left(Y^{2} e^{\left.-c(\log Y)^{1 / 2}\right),}\right.
$$




$$
\sum_{\substack{(\omega)_{1} \\ \omega \varepsilon_{0} \\ N(\omega)<Y}} N(\omega) \log N(\omega)=\frac{q}{2 h} \frac{Y^{2}}{2}+O\left(Y^{2} e^{\left.-c(\log Y)^{1 / 2}\right)} .\right.
$$

With the abbreviation $Y=x x^{\prime}$ we get from (22a)

and hence

$$
\begin{aligned}
H=\frac{1}{\log \eta} \sum_{\substack{(\omega)_{1} \\
\omega \varepsilon=0 \\
N(\omega)<Y}}\{Y(\log Y-2)+N(\omega)(\log Y+2)-Y \log N(\omega) \\
-N(\omega) \log N(\omega)\}
\end{aligned}
$$

$$
\begin{aligned}
H= & \frac{1}{\log \eta} \frac{q}{2 h}\left\{Y(\log Y-2) \int_{2}^{Y} \frac{d t}{\log t}+(\log Y+2) \int_{2}^{Y} \frac{t d t}{\log t}-\frac{3 Y^{2}}{2}\right\} \\
& +O\left(Y^{2} e^{-c(\log Y) 1 / 2}\right),
\end{aligned}
$$

and finally from (27), in view of (18),

$$
P_{1}\left(x, x^{\prime}\right)=\sum_{0-3 \omega-3 x}(x-\omega)\left(x^{\prime}-\omega^{\prime}\right)
$$

$$
\begin{aligned}
= & \frac{1}{2 h \log \epsilon}\left\{Y(\log Y-2) \int_{2}^{Y} \frac{d t}{\log t}+(\log Y+2) \int_{2}^{Y} \frac{t d t}{\log t}-\frac{3 Y^{2}}{2}\right\} \\
& +O\left(Y^{2} e^{-c(\log Y) 1 / 2}\right), \quad Y=x x^{\prime} .
\end{aligned}
$$

3. Passing from $P_{1}\left(x, x^{\prime}\right)$ to $P_{0}\left(x, x^{\prime}\right)$

Let $\delta$ be a positive number, $\delta<x$, which may be at our disposal until later. We have on one hand

$$
P_{1}\left(x+\delta, x^{\prime}\right)-P\left(x, x^{\prime}\right)=\delta \sum_{\substack{0<\omega<x \\ 0<\omega^{\prime}<x^{\prime}}}\left(x^{\prime}-\omega^{\prime}\right)+\sum_{\substack{x \leqq \omega<x+\delta \\ 0<\omega^{\prime}<x^{\prime}}}(x+\delta-\omega)\left(x^{\prime}-\omega^{\prime}\right)
$$

and on the other hand from (33)

$$
\begin{aligned}
& 2 h \log \epsilon\left(P_{1}\left(x+\delta, x^{\prime}\right)-P_{1}\left(x, x^{\prime}\right)\right) \\
&=\left((x+\delta) x^{\prime}\left(\log (x+\delta) x^{\prime}-2\right)-x x^{\prime}\left(\log x x^{\prime}-2\right)\right) \int_{2}^{x x^{\prime}} \frac{d t}{\log t} \\
&+(x+\delta) x^{\prime}\left(\log (x+\delta) x^{\prime}-2\right) \int_{x x^{\prime}}^{(x+\delta) x^{\prime}} \frac{d t}{\log t} \\
&+\left(\log (x+\delta) x^{\prime}-\log x x^{\prime}\right) \int_{2}^{x x^{\prime}} \frac{t d t}{\log t} \\
&+\left(\log (x+\delta) x^{\prime}+2\right) \int_{x x^{\prime}}^{(x+\delta) x^{\prime}} \frac{t d t}{\log t}-\frac{3}{2} \delta x^{\prime}\left(2 x x^{\prime}+\delta x^{\prime}\right) \\
&+O\left(\left(x x^{\prime}\right)^{2} e^{-c\left(\log x x^{\prime}\right)^{1 / 2}}\right) .
\end{aligned}
$$


We give now an estimate of the second sum on the right-hand side in (34),

$$
\sum_{\substack{x \leqq \omega<x+\delta \\ 0<\omega^{\prime}<x^{\prime}}}(x+\delta-\omega)\left(x^{\prime}-\omega^{\prime}\right)=O\left(\delta x^{\prime} \sum_{\substack{x \leqq \mu<x+\delta \\ 0<\mu^{\prime}<x^{\prime}}} 1\right),
$$

where $\mu$ runs through all integers in the assigned rectangle. The integers forming a point lattice, their number is of the order of the rectangle's area, plus an error-arising from the boundaries. But as the boundaries of the rectangle of one small side $\delta$ and one long side $x^{\prime}$ are relatively long, we have to transform the rectangle into a more suitable shape. In fact, there are as many integers in

$$
x \leqq \mu<x+\delta, \quad 0<\mu^{\prime}<x,
$$

as in

$$
x \eta^{l} \leqq \mu<(x+\delta) \eta^{l}, \quad 0<\mu^{\prime}<x^{\prime} \eta^{-l},
$$

and we can choose the exponent $l$ in such a way as to have $\delta \eta^{l}$ and $x^{\prime} \eta^{-l}$ of the same order, i.e.,

$$
\delta \eta^{l}=O\left(\left(\delta x^{\prime}\right)^{1 / 2}\right), \quad x^{\prime} \eta^{-l \cdot}=O\left(\left(\delta x^{\prime}\right)^{1 / 2}\right) .
$$

Then the length of the boundary of the rectangle is $O\left(\left(\delta x^{\prime}\right)^{1 / 2}\right)$, and we have

$$
\sum_{\substack{x \leqq \mu<x+\delta \\ 0<\mu^{\prime}<x^{\prime}}} 1=O\left(\delta x^{\prime}+\left(\delta x^{\prime}\right)^{1 / 2}+1\right)
$$

and hence

$$
\sum_{\substack{x \leqq \omega<x+\delta \\ 0<\omega<x^{\prime}}}(x+\delta-\omega)\left(x^{\prime}-\omega^{\prime}\right)=O\left(\delta^{2} x^{\prime 2}\right)+O\left(\delta^{3 / 2} x^{\prime 3 / 2}\right)+O\left(\delta x^{\prime}\right) .
$$

Moreover we have

and

$$
\begin{aligned}
\log (x+\delta) x^{\prime}-\log x x^{\prime}=\log \left(1+\frac{\delta}{x}\right)=\frac{\delta}{x}+o\left(\frac{\delta^{2}}{x^{2}}\right) \\
\begin{aligned}
(x+\delta) x^{\prime} & \log (x+\delta) x^{\prime}-x x^{\prime} \log x x^{\prime} \\
= & x x^{\prime}\left(\log (x+\delta) x^{\prime}-\log x x^{\prime}\right)+\delta x^{\prime} \log (x+\delta) x^{\prime} \\
& =\delta x^{\prime}+O\left(\frac{\delta^{2} x^{\prime}}{x}\right)+\delta x^{\prime} \log x x^{\prime}+O\left(\frac{\delta^{2} x^{\prime}}{x}\right) \\
& =\delta x^{\prime}\left(1+\log x x^{\prime}\right)+O\left(\frac{\delta^{2} x^{\prime}}{x}\right)
\end{aligned}
\end{aligned}
$$




$$
\begin{aligned}
\int_{x x^{\prime}}^{(x+\delta) x^{\prime}} \frac{d t}{\log t} & =\frac{\delta x^{\prime}}{\log x x^{\prime}}+O\left(\delta x^{\prime}\left(\frac{1}{\log x x^{\prime}}-\frac{1}{\log (x+\delta) x^{\prime}}\right)\right) \\
& =\frac{\delta x^{\prime}}{\log x x^{\prime}}+O\left(\frac{\delta^{2} x^{\prime}}{x\left(\log x x^{\prime}\right)^{2}}\right)
\end{aligned}
$$

and similarly

$$
\begin{aligned}
\int_{x x^{\prime}}^{(x+\delta) x^{\prime}} \frac{t d t}{\log t} & =\delta x^{\prime} \frac{x x^{\prime}}{\log x x^{\prime}}+O\left(\delta x^{\prime}\left(\frac{(x+\delta) x^{\prime}}{\log (x+\delta) x^{\prime}}-\frac{x x^{\prime}}{\log x x^{\prime}}\right)\right) \\
& =\delta x^{\prime} \frac{x x^{\prime}}{\log x x^{\prime}}+O\left(\frac{\delta^{2} x^{\prime 2}}{\log x x^{\prime}}\right) .
\end{aligned}
$$

The equations (34) to (40) give, after division by $\delta$ and after due simplifications,

$$
\begin{aligned}
2 h \log \in Q\left(x, x^{\prime}\right)= & 2 h \log \epsilon \sum_{\substack{0<\omega<x \\
0<\omega^{\prime}<x}}\left(x^{\prime}-\omega^{\prime}\right) \\
= & x^{\prime}\left(\log x x^{\prime}-1\right) \int_{2}^{x x^{\prime}} \frac{d t}{\log t}+\frac{1}{x} \int_{2}^{x x^{\prime}} \frac{t d t}{\log t} \\
& -x x^{\prime 2}+O\left(\delta x^{\prime 2}\right)+O\left(\delta^{1 / 2} x^{\prime 3 / 2}\right)+O\left(x^{\prime}\right) \\
& +O\left(\frac{\left(x x^{\prime}\right)^{2} e^{-c\left(\log x x^{\prime}\right) 1 / 2}}{\delta}\right) .
\end{aligned}
$$

The same process that we applied to $x$ is now to be used with respect to $x^{\prime}$. Let $\delta^{\prime}$ be positive, $\delta^{\prime}<x^{\prime}$. We have on one hand

$$
\begin{aligned}
Q\left(x, x^{\prime}+\delta^{\prime}\right)-Q\left(x, x^{\prime}\right) & =\sum_{\substack{0<\omega<x \\
0<\omega^{\prime}<x^{\prime}+\delta^{\prime}}}\left(x^{\prime}+\delta^{\prime}-\omega^{\prime}\right)-\sum_{\substack{0<\omega<x \\
0<\omega^{\prime}<x^{\prime}}}\left(x^{\prime}-\omega^{\prime}\right) \\
& =\delta^{\prime} \sum_{\substack{0<\omega<x \\
0<\omega^{\prime}<x^{\prime}}} 1+\sum_{\substack{0<x^{\prime} \\
x^{\prime} \leqq \omega^{\prime}<x^{\prime}+\delta^{\prime}}}\left(x^{\prime}+\delta^{\prime}-\omega^{\prime}\right) .
\end{aligned}
$$

Here

$$
\sum_{\substack{0<\omega<x \\ x^{\prime} \leqq \omega^{\prime}<x^{\prime}+\delta^{\prime}}}\left(x^{\prime}+\delta^{\prime}-\omega^{\prime}\right)=O\left(\delta_{\substack{0<\mu<x \\ \delta^{\prime} \leqq \mu^{\prime}<x^{\prime}+\delta^{\prime}}} 1\right)=O\left(\delta^{\prime 2} x+\delta^{\prime 3 / 2} x^{1 / 2}+\delta^{\prime}\right)
$$

in analogy to our former argument. From these equations and from (41) we deduce on the other hand 


$$
\begin{aligned}
& 2 h \log \epsilon \cdot \delta^{\prime} \sum_{\substack{0<\omega<x \\
0<\omega^{\prime}<x^{\prime}}} 1 \\
& =\left(\left(x^{\prime}+\delta^{\prime}\right)\left(\log x\left(x^{\prime}+\delta^{\prime}\right)-1\right)-x^{\prime}\left(\log x x^{\prime}-1\right)\right) \int_{2}^{x x^{\prime}} \frac{d t}{\log t} \\
& \quad+\left(x+\delta^{\prime}\right)\left(\log x\left(x^{\prime}+\delta^{\prime}\right)-1\right) \int_{x x^{\prime}}^{x\left(x^{\prime}+\delta^{\prime}\right)} \frac{d t}{\log t}+\frac{1}{x} \int_{x x^{\prime}}^{x\left(x^{\prime}+\delta^{\prime}\right)} \frac{t d t}{\log t} \\
& \quad-x \delta^{\prime}\left(2 x^{\prime}+\delta^{\prime}\right)+O\left(\delta^{\prime 2} x\right)+O\left(\delta^{\prime 3 / 2} x^{1 / 2}\right)+O\left(\delta^{\prime}\right)+O\left(\delta x^{\prime 2}\right) \\
& \quad+O\left(\delta^{1 / 2} x^{\prime 3 / 2}\right)+O\left(x^{\prime}\right)+O\left(\delta^{-1}\left(x x^{\prime}\right)^{2} e^{\left.-c\left(\log x x^{\prime}\right) 1^{1 / 2}\right) .}\right.
\end{aligned}
$$

If we make use of (38), (39), (40) after replacing $x, \delta$ by $x^{\prime}, \delta^{\prime}$ and vice versa, we get from (42) after division by $\delta^{\prime}$ and some easy calculations

$$
\begin{aligned}
2 h \log \epsilon & \sum_{\substack{0<\omega<x \\
0<\omega^{\prime}<x^{\prime}}} 1=\log x x^{\prime} \int_{2}^{x x^{\prime}} \frac{d t}{\log t}-x x^{\prime}+O\left(\delta^{\prime} x\right)+O\left(\delta^{\prime 1 / 2} x^{1 / 2}\right)+O(1) \\
& +O\left(\frac{\delta}{\delta^{\prime}} x^{\prime 2}\right)+O\left(\frac{\delta^{1 / 2} x^{\prime 3 / 2}}{\delta^{\prime}}\right)+O\left(\frac{x^{\prime}}{\delta^{\prime}}\right)+O\left(\frac{\left(x x^{\prime}\right)^{2}}{\delta \delta^{\prime}} e^{-c\left(\log x x^{\prime}\right) 1 / 2}\right) .
\end{aligned}
$$

Now we put

$$
\delta=x e^{-(c / 2)\left(\log x x^{\prime}\right) 1 / 2}, \quad \delta^{\prime}=x^{\prime} e^{-(c / 4)\left(\log x x^{\prime}\right)^{1 / 2}},
$$

and have then

$$
\sum_{\substack{0<\omega<x \\ 0<\omega^{\prime}<x^{\prime}}} 1=\frac{1}{2 h \log \epsilon}\left(\log x x^{\prime} \int_{2}^{x x^{\prime}} \frac{d t}{\log t}-x x^{\prime}\right)+O\left(x x^{\prime} e^{-(c / 4)\left(\log x x^{\prime}\right) 1 / 2}\right) .
$$

As we find by partial integration

$$
\log Y \int_{2}^{Y} \frac{d t}{\log t}=Y+\log Y \int_{2}^{Y} \frac{d t}{(\log t)^{2}}+O(\log Y),
$$

we have finally, with $c$ in a new meaning,

(43) $P_{0}\left(x, x^{\prime}\right)=\sum_{\substack{0<\omega<x \\ 0<\omega^{\prime}<x^{\prime}}} 1=\frac{1}{2 h \log \epsilon} \log x x^{\prime} \int_{2}^{x x^{\prime}} \frac{d t}{(\log t)^{2}}+O\left(x x^{\prime} e^{-c\left(\log x x^{\prime}\right) 1 / 2}\right)$,

which is the special case $a=(1)$ of the theorem on page 76 of my paper "K."

University of Pennsylvania,

Philadelphia, Pa. 